\title{
Quality-of-life outcomes in patients with gynecologic cancer with colostomy and effectiveness of psychologist consultation
}

\begin{abstract}
Objective: If the female genital cancer is associated with colostomy creates dilemma. We explored the impact of an integrative colostomy and oncology therapeutic regimen on quality-of-life (QOL) outcomes in women with gynecological cancer whit colostomy.

Patients and methods: Study on 102 patients selected as randomize from patients referred to oncology and surgical clinic all of patients consultation with psychologist. Quality of life and wellbeing, at baseline at a 6-12-week follow-up were assessment.

Results: Of 102 patients referred to oncologic clinic and surgery, 83 patients were fatigue $(81.8 \%)$, gastrointestinal symptoms 66 patients $(64.6 \%)$, pain and neuropathy 55 patients $(54.5 \%)$, and emotional distress 46 patients $(45.5 \%)$. Fatigue scores improved by a mean when consultant with psychologist done. Differences in quality of life were considered significant at $P<0.05$.

Conclusion: If conditions provide patient comfort and consultant with psychologist the gynecological cancer during chemotherapy may reduce cancer-related fatigue and improve other QOL outcomes.
\end{abstract}

Keywords: oncology; surgery; psychology; quality-of-life
Volume 7 Issue 6 - 2017

\author{
Seyed Reza Mousavi,' Seyed Mahdi Mousavi, ${ }^{2}$ \\ Mohammad Esmaeel Akbari ${ }^{3}$ \\ 'Professor of surgical oncologist, Shohada Medical Center and \\ Cancer Research Center, Shaheed Beheshti University Medical \\ Sciences, Iran \\ ${ }^{2}$ Psychologist, Cancer Research Center, Shohada Medical Center, \\ Beheshti University Medical Sciences, Iran \\ ${ }^{3}$ Professor of surgical oncologist, Shohada Medical Center and \\ Head of Cancer Research Center, Shaheed Beheshti University \\ Medical Sciences, Iran
}

\begin{abstract}
Correspondence: Seyed Reza Mousavi, Shohada Medica Center and Cancer Research Center, Shaheed Beheshti University Medical Sciences, Tajrish, Tehran, Iran, Tel 9821 22721 I44, Email prof.srmousavi@gmail.com
\end{abstract}

Received: January 30, 2017 | Published: April 17, 2017

\section{Introduction}

Quality of life with colostomy despite difficult, colorectal cancer is the most common cause of intestinal stormy surgery. ${ }^{1,2}$ Using stoma, either permanent or temporary, greatly reduces the patient's quality of life (QOL) ${ }^{3,4}$ Reasons for malignancy rectum surgery are varied, but the most common causes are colon and rectum cancer. ${ }^{5,6}$

Treatment of gynecologic malignancies often involves a multimodality approach and is associated with high morbidity and changes in bowel, bladder, hormonal, sexual, and reproductive function, many of which can cause emotional and psychosocial in addition to physical distress. ${ }^{7,8}$ In such circumstances, it is worthwhile to assess quality of life in the evaluation of the outcomes of various therapeutic procedures along with their final impact on patients' lives. ${ }^{1}$ Quality of life for gynecologic cancer patients tends to be at its worst between time of diagnosis and completion of radiotherapy, especially when they have colostomy. ${ }^{7}$ Therefore, it is important to consultant with the psychotherapist during the chemotherapy and follow for long time under psychotherapy for the emotional and functional components of quality of life in gynecologic cancer and rectum involvement patients while on active treatment, a critical window for additional clinician support. Previous studies suggest that psychosocial interventions for cancer patients in the acute setting improve patients' psychological well-being. ${ }^{9,10}$ Palliative care is applicable early in the course of illness, in conjunction with other therapies that are intended to prolong life, such as chemotherapy or radiation therapy, and includes those investigations needed to better understand and manage distressing clinical complications. ${ }^{11}$ Because radiation and chemotherapy and surgery for these patients has been associated with risk of poor adjustment, our objective was to identify the trajectory of patients' quality of life during and after treatment, particularly the role of psychosocial interactions on patient quality of life. We believe that it is consulting with a psychologist during and after treatment significant impact on quality of life.

\section{Patients and methods}

At first, we inform consent was obtained from all patients. The study was designed as a prospective registry protocol-based preference study, evaluating the impact of an positive consultant psychologist intervention on patients' concerns and well-being, including symptoms induced by after surgery and chemotherapy and radiotherapy . Eligible participants were patients diagnosed with gynecological cancer invasion to rectum and colon aged $\geq 30$ years who were undergoing therapy at Shohada Medical Center north of Tehran, between March 2008 and May 2015.

\section{Role of cancer research center integrative}

This center service provides patients program of pre and post cancer surgery managements and patients with radiotherapy and chemotherapy gives, within a conventional oncology clinic. Hospitalbased surgical treatments are provided, as well as in-patient treatments of chemotherapy-radiotherapy and palliative care.

The first goal was established with three main, the first of which was the assessment of patients' con-cerns, physical symptoms, and effect on quality of life, second psychological problems, and third socioeconomic relations.

We have program pre operative evaluation all probably therapies and first visit by a consultant psychologist then obtained inform consent and patients admitted to the gynecology and surgery wards. Intra operation two teams gynecologist and colorectal or cancer surgeon sharing the operation. After surgery and discharged from hospital we see all patients for probably physical symptom and evaluate the general conditions. Post - operative visit by psychologist just one month and before begin chemo or radiotherapy and serial visit along duration is done. The important subject is continuing visit by psychologist for long of time. 


\section{Data analysis}

All data were entered in a database and analyzed with SPSS v.13.0 software. In accordance with the study objectives, patients follow - up and consultation with psychologist were used for different subscales and for the entire patients. For this procedure, the overall quality of life score of the patients was treated as the dependent variable. Demographic data and variables relating to changes in life style were entered in these patients. Differences in quality of life were considered significant at $P<0.05$.

\section{Results}

Of 102 patients referred to two clinics oncology and surgery, 83 patients were fatigue $(81.8 \%$ ), gastrointestinal symptoms 66 patients (64.6\%), pain and neuropathy 55 patients $(54.5 \%)$, and emotional distress 46 patients $(45.5 \%)$. Fatigue scores improved by a mean when consultant with psychologist done. Differences in quality of life were considered significant at $P<0.05$.

The mean age of the patients was $53.5 \pm 12.3$ years. According to the patients' reports, $83.3 \%, 82.4 \%$, and $48 \%$ of stoma patients had been forced to change their job, diet, and clothing style, respectively. Considering the psychological implications after colostomy surgery, approximately $63 \%(n=64)$ had feelings of depression. Having problem with the location of colostomy was reported by $41.2 \%$ ( $n=$ 42) of the study sample.

While the majority of patients $(83.4 \%)$ reported being sexually active before colostomy surgery, only $31.3 \%$ resumed sexual activity after surgery. 32 patients $(31.4 \%)$ were reported being satisfied with sexual activities.

According to the reports of most patients, it took them at least 6 months to feel comfortable with the daily care and diet. The majority of colostomy patients had no restrictions on the consumption of specific foods such as carbonated beverages, dairy products, fruits, snacks, and vegetables.

The mean score for the overall quality of life in colostomy patients was $7.47 \pm 0.9$. The best outcomes were found for the therapy by psychologist subscale and the without therapy subscale scored the lowest (Table1).

Table I Mean and standard deviation

\begin{tabular}{lll}
\hline Subscale & Mean & Standard deviation \\
\hline Physical & 7.26 & 1.11 \\
Psychological & 7.86 & 1.08 \\
Other (spiritual, social...) & 7.31 & 1.12 \\
\hline
\end{tabular}

\section{Conclusion}

Studies shown colostomy worsened quality of life, and when consultation with psychologists during therapy may be effective in good results. According to the results, most patients reported that they have lost their sexual activity after stoma surgery. The findings of the current study are consistent with other studies. ${ }^{1,11-15}$ Physical problem especially due to colostomy is very important for patients including irritated peristomal skin, odor, and noise from the appliance mentioned by participants, have been reported by other investigators. ${ }^{16-18}$ Outpatient palliative care consultation has been associated with decreased symptom burden as measured by the Edmonton Symptom Assessment Scale (ESAS). ${ }^{19}$ Outpatient palliative medicine consultation has been reported to be cost-effective for patients with cervical and ovarian cancer. ${ }^{20,21}$ Additionally, the gold standard for these patients should include measure of quality-of-life and awareness for families, which they may expect full recovery. Future research is needed to validate whether there are improvements in patient and family quality of life following palliative medicine consultation (as psychologist) with prospective testing of quality of life assessments in patients with gynecologic malignancies with colostomy. Attention towards the impact of psychosocial distress on quality of life has been increasingly recognized as emerging research suggests that methods that screen for and address distress improves quality of life, but may also be associated with improved cancer outcomes. ${ }^{22,23}$ Patientreported health-related quality of life may be useful for identifying patients who can benefit from additional support before and during adjuvant therapy. Particularly for gynecologic patients receiving radiotherapy, additional psychosocial and emotional support before and during treatment may be warranted. ${ }^{24}$ One of important subject in medical management after surgery are medical judgments about the disease and selection of treatment(s) consider these variables as well as many others, including the aggressiveness and predictability of the disease, the medical morbidity and mortality of the therapy, overall cure rate, and physician/institution experience in treating the disease. ${ }^{25}$ Some researchers studying psychological interventions for cancer patients were detailed and good outcome. Therefore, other articles can provide discussions of many impediments, such as the training circumstances, and may have resulted in relatively few psychologists' having a primary focus on cancer. ${ }^{26,27}$

A special category of information that gynecologic oncology patients may need is that regarding sexual functioning and the strategies for preventing sexual difficulties. As indicated previously, radiation therapy appears to result in sexual disruption. ${ }^{28}$ Dyspareunia, the most frequent complaint, after radiotherapy and treatments, receiving only external therapy reported relieve this symptom too. when need psychological assistance during cancer treatments a brief therapy or crisis intervention may be beneficial, and psychotropic medication also may be warranted for the level of depression or anxiety many cancer patients experience. ${ }^{29}$ Although the type of treatment received is influential, individual differences in anxiety may also help to explain fluctuations in patients' emotional status and therefore, least distressed before treatment often show the worst adaptation after treatment. ${ }^{30,31}$ There is strong evidence that cancer and its different forms of treatment can have consequences for a survivor's sexual function. This has been most studied in ovarian, bladder and cervical cancer. There is also evidence to suggest that symptoms that accompany treatment-induced menopause are distressing. Psychooncology now has a recognized role within the oncologic community through clinical care, research, and training as it relates to prevention of cancer through lifestyle changes, evaluation of quality of life, symptom control, palliative care and survivorship. ${ }^{32}$

Pelvic exenteration is considered for some women with recurrent cervical cancer. It is a disfiguring operation, involving removal of the uterus, tubes, ovaries, urinary bladder, rectum, and vagina. Clinical reports have portrayed lengthy postoperative recovery, residual affective disruption, and significant disruption or, more likely, cessation of sexual activity. ${ }^{33,34}$

Pain in cancer patients is a complex issue; Cancer-related pain may result from the cancer itself or from associated treatments. ${ }^{35}$ With a lot of evidence to say that pain due to cancer, obviously has an impact on quality of life and persistent pain will lead to poorer general health, physical, role and social functioning in cancer survivors. ${ }^{36}$ 
As successful as chemotherapy, radiation, and surgery may be for some patients with cancer, the benefits of those therapies usually do not come easily. One well known side effect of cancer treatment is depression, and practitioners have many resources to help their patients cope with it. Depression is the only psychological disorder with that distinction. ${ }^{37,38}$ In general, these patients need to have a teamwork for the desired result.

\section{Funding}

None.

\section{Acknowledgements}

We sincerely co-operate Department of Surgery and Cancer Research Center thanks our dedicated staff.

\section{Conflicts of interests}

The authors declare that there is no conflict of interest.

\section{References}

1. Carlsson E, Berndtsson I, Hallen AM, et al. Concerns and quality of life before surgery and during the recovery period in patients with rectal cancer and an ostomy. $J$ Wound Ostomy Continence Nurs. 2010;37(6):654-661.

2. Marquis P, Marrel A, Jambon B. Quality of life in patients with stomas: The Montreux Study. Ostomy Wound Manage. 2003;49(2):48-55.

3. Taylor C, Morgan L. Quality of life following reversal of temporary stoma after rectal cancer treatment. Eur J Oncol Nurs. 2011;15(1):5966.

4. Siassi M, Hohenberger W, Losel F, et al. Quality of life and patient's expectations after closure of a temporary stoma. Int $J$ Colorectal Dis. 2008;23(12):1207-1212.

5. Krouse RS, Grant M, Rawl SM, et al. Coping and acceptance: The greatest challenge for veterans with intestinal stomas. J Psychosom Res. 2009;66(3):227-232.

6. Smith DM, Loewenstein G, Rozin P, et al. Sensitivity to disgust, stigma, and adjustment to life with a colostomy. $J$ Res Pers. 2007;41(4):787803.

7. Pearman T. Quality of life and psychosocial adjustment in gynecologic cancer survivors. Health Qual Life Outcomes. 2003;20: 1-33.

8. Yavas G, Dogan NU, Yavas C, et al. Prospective assessment of quality of life and psychological distress in patients with gynecologic malignancy: a 1-year prospective study. Int J Gynecol Cancer. 2012;22(6):1096-101.

9. Goerling U, Tagmat D, Muffler E. Practice and effectiveness of outpatient psycho-oncological counseling for cancer patients. $J$ Cancer. 2010;23(1):112-119.

10. Miller JJ, Frost MH, Rummans TA, et al. Role of a medical social worker in improving quality of life for patients with advanced cancer with a structured multidisciplinary intervention. J Psychosoc Oncol. 2007;25(4):105-119.

11. Sepulvada C, Marlin A, Yoshida T, et al. Palliative care: the World Health Organization's global perspective. J Pain Symptom Manage. 2002;24(2):91-96.

12. Marquis P, Marrel A, Jambon B. Quality of life in patients with stomas: The Montreux Study. Ostomy Wound Manage. 2003;49(2):48-55.

13. Dabirian A, Yaghmaei F, Rassouli M,et al. Quality of life in ostomy patients: A qualitative study. Patient Prefer Adherence. 2010;21(5):1-5.
14. Krouse R, Grant M, Ferrell B, et al. Quality of life outcomes in 599 cancer and non-cancer patients with colostomies. J Surg Res. 2007;138(1):79-87.

15. Aronovitch SA, Sharp R, Harduar-Morano L.Quality of life for patients living with ostomies: Influence of contact with an ostomy nurse. $J$ Wound Ostomy Continence Nurs. 2010;37(6):649-653.

16. Richbourg L, Thorpe JM, Rapp CG. Difficulties experienced by the ostomate after hospital discharge. J Wound Ostomy Continence Nurs. 2007;34(1): 70-79.

17. Mitchell KA, Rawl SM, Schmidt CM, et al. Demographic, clinical, and quality of life variables related to embarrassment in veterans living with an intestinal stoma. $J$ Wound Ostomy Continence Nurs. 2007;34(5): 524-532.

18. Lynch BM, Hawkes AL, Steginga SK, et al.. Stoma surgery for colorectal cancer: A population-based study of patient concerns. J Wound Ostomy Continence Nurs. 2008;35(4):424-428.

19. Ruskin R, Rabow M, Alen I, et al. Outpatient palliative care consultation is associated with a decrease in symptom burden for women with gynecologic malignancies. Gynecol Oncol. 2013;31(1):255.

20. Lowery W, Lowery A, Barnett J, et al. Cost-effectiveness of early palliative care intervention in recurrent platinum-resistant ovarian cancer. Gynecol Oncol. 2013;130(3):426-430.

21. Phippen N, Leath C, Miller C, et al. Is a home-based palliative care treatment strategy preferable to standard chemotherapy in recurrent cervical cancer?. Gynecol Oncol. 2013;130(1):e30.

22. Andersen B, Thornton L, Shapiro C. Biobehavioral, immune, and health benefits following recurrence for psychological intervention participants. Clin Cancer Res. 2010;16(12):3270-3278.

23. Giese-Davis J, Collie K, Rancourt K, et al. Decrease in depression symptoms is associated with longer survival in patients with metastatic breast cancer: a secondary analysis. J Clin Oncol. 2010;29(4):413-420.

24. Kirchheiner K, Czajka-Pepl A, Ponocny-Seliger E, et al. Posttraumatic stress disorder after high-dose-rate brachytherapy for cervical cancer with 2 fractions in 1 application under spinal/epidural anesthesia: incidence. Int J Radiat Oncol Biol Phys. 2014;89(2):260-267

25. Osteen RT, Steele GD, Menck HR, et al. Regional differences in surgical management of breast cancer. $C a-A$ Cancer Journal for Clinicians. 1922;42(1):39-43

26. Andersen BL, Beck G, Ouelette-Kobasa S, et al. Directions for a psychology research agenda in cancer. Health Psychology. 1989;8(6):753-760.

27. Burish TG. Progress in psychosocial and behavioral cancer research: The need for enabling strategies. Cancer. 1991;67(3 Suppl): 860-864.

28. Andersen BL, Hacker NF. Psychosexual adjustment of gynecologic oncology patients: a proposed model for future investigation. Gynec Oncol. 1983;15(2):214-223.

29. Derogatis LR, Feldstein M, Morrow G, et al.A survey of psychotropic drug prescriptions in an oncology population. Cancer. 1979;44(5):19191929.

30. Andersen BL, Tewfik HH. Psychological reactions to radiation therapy: reconsideration of the adaptive aspects of anxiety. J pers soc Psychol. 1985;48(4):1024-1032.

31. Andersen BL, Karlsson JA, Anderson BA, et al. Anxiety and cancer treatment: response to stressful radiotherapy. Health Psychol. 1984;3(6):535-551.

32. Jimmie C. Holland. Psychological Care of Patients: Psycho-Oncology's Contribution. J Clin Oncol. 2003;21(23 Suppl):253s-265s. 
33. Brown RS, Haddox V, Posada A,et al. Social and psychological adjustment following pelvic exenteration. Am J Obstet Gynecol. 1972;114(2): 162-171.

34. Vera MI. Quality of life following pelvic exenteration. Gynecol Oncol. 1981;12(3):355-366.

35. Grond S, Zech D, Diefenbach C, et al. Assessment of cancer pain: a prospective evaluation in 2266 cancer patients referred to a pain service. Pain.1996;64(1):107-114.
36. Green CR, Hart-Johnson T, Loeffler DR. Cancer-related chronic pain: examining quality of life in diverse cancer survivors. Cancer. 2011;117(9):1994-2003.

37. Satin JR, Linden W, Phillips MJ. Depression as a predictor of disease progression and mortality in cancer patients: a meta-analysis. Cancer. 2009;115(22):5349-5361. 